\title{
Subclinical hypothyroidism increased the risk of placental abruption and poor neonatal outcomes
}

Casey BM, Dashe JS, Wells CE, et al. Subclinical hypothyroidism and pregnancy outcomes. Obstet Gynecol 2005;105:239-45.

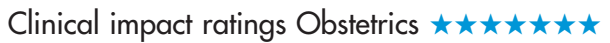

\section{Is subclinical hypothyroidism (SH) a risk factor for poor pregnancy and neonatal outcomes?}

\section{METHODS}

-

Design: cohort study with follow up at birth.

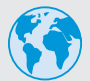

Setting: a county hospital in Dallas, Texas, USA.

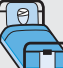

Patients: 16093 pregnant women (mean age 25.5 y) enrolled for prenatal care at $\leqslant 20$ weeks of gestation who had thyroid screening.

Risk factors: SH (elevated serum thyroid stimulating hormone $[\mathrm{TSH}]$ concentration $\geqslant 97.5^{\text {th }}$ percentile corrected for gestationa age: range $2.74-5.09 \mathrm{mU} / \mathrm{l}$ and a normal serum free thyroxine concentration $>0.680 \mathrm{ng} / \mathrm{dll}$ ). Thyroid function tests were done using chemiluminescent assays for TSH and free thyroxine (Immulite 2000 Analyzer [Diagnostic Products Corporation, Los Angeles, CA]). Women were retrospectively identified as having $\mathrm{SH}$

Outcomes: pregnancy outcomes: gestational hypertension (intrapartum systolic blood pressure [BP] $\geqslant 140 \mathrm{~mm} \mathrm{Hg}$ or diastolic $B P \geqslant 90 \mathrm{~mm} \mathrm{Hg}$ ), and severe preeclampsia ( $\geqslant 1$ of $B P$

$>160 / 110 \mathrm{~mm} \mathrm{Hg}$, serum creatinine $>1.0 \mathrm{mg} \%$, platelet coun $<100000 / \mu \mathrm{l}$, serum aspartate aminotransferase concentration at least twice the upper normal value, persistent headache or scotomata, or significant proteinuria. Neonatal outcomes: birth weight, preterm birth (gestational age of $\leqslant 34 \mathrm{wks}$ at delivery) other conditions consistent with prematurity, and major malformations.

\section{MAIN RESULTS}

404 women had $\mathrm{SH}$ with TSH concentrations ranging from 2.74 to $>75 \mathrm{mU} / \mathrm{l}$. More women with $\mathrm{SH}$ were $\geqslant 35$ years of age than those without SH $(11 \% \vee 7 \%$, respectively; $\mathrm{p}=0.009)$. Pregnancy outcomes. Women with $\mathrm{SH}$ were 3 times more likely to have a pregnancy complicated by placental abruption than those without SH (table).

For correspondence: Dr B M Casey, University of Texas Southwestern Medical Center, Dallas, TX, USA. brian.casey@utsouthwestern.edu

Source of funding: no external funding.

Pregnancy and neonatal outcomes of pregnant women with subclinical hypothyroidism (SH) compared with women without $\mathrm{SH}^{*}$

\begin{tabular}{ll}
\hline Outcomes & Relative riskt $(95 \% \mathrm{Cl})$ \\
\hline Placental abruption & $3.0(1.1$ to 8.2$)$ \\
Preterm birth & $1.8(1.1$ to 2.9$)$ \\
Admission to the NICN & $1.8(1.1$ to 2.9$)$ \\
Respiratory distress & $1.8(1.0$ to 3.3$)$ \\
\hline
\end{tabular}

* $\mathrm{NICN}=$ neonatal intensive care nursery; $\mathrm{Cl}$ and other abbreviations defined in glossary.

+Unadjusted, but significant differences persisted after adjustment for maternal age, race, and placental abruption (Hosmer-Lemeshow goodness of fit, $p=0.97$ ).
Preterm birth was close to 2 fold higher in women with SH (table). Neonatal outcomes. Birth weight of infants delivered by women with $\mathrm{SH}$ did not differ from those without SH (mean $3.317 \mathrm{~g} v 3.367 \mathrm{~g}$, $\mathrm{p}=0.081$ ). Admission to the neonatal intensive care nursery (NICN) and respiratory distress were twice as likely in infants delivered by women with $\mathrm{SH}$ (table). The groups did not differ for major malformations $(0.5 \% \vee 1.0 \%, \mathrm{p}=0.231)$, fetal death $(0.5 \% v 0.5 \%$, $\mathrm{p}=0.995)$, or neonatal death $(0.5 \% \vee 0.2 \%, \mathrm{p}=0.295)$.

\section{CONCLUSION}

Subclinical hypothyroidism was associated with increased risk of having a pregnancy complicated by placental abruption, preterm birth, admission to the neonatal intensive care nursery, and respiratory distress.

\section{Commentary}

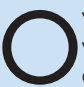

vert maternal thyroid disease occurs in approximately $1-2 \%$ whereas $\mathrm{SH}$ is thought to occur in $2-3 \%$ of women. ${ }^{1}$ The health effects of untreated or inadequately treated hypothyroidism are well documented and include miscarriage, placental abruption, intrauterine growth restriction, perinatal and postnatal morbidity, and neuropsychological impairments in children. However, the effects of SH on pregnancy outcome are much less clear.

Using a prospective study design of $>16000$ young pregnant women (mean age $25.5 \mathrm{y}$ ), the study by Casey et al showed that $\mathrm{SH}$ in the second trimester is associated with a significantly increased risk of placental abruption, preterm birth, admission to the NICN, and respiratory distress. In an effort to identify and treat thyroid disease in pregnancy and to combat associated childhood neuropsychological impairments, the issue of prenatal thyroid screening has been suggested as a public health policy in recent years. Results of this study will add to the ongoing thyroid screening debate and raise a number of important questions: What stage in pregnancy should pregnant women be evaluated for thyroid disease? What serum level of TSH triggers the need for treatment? Does $\mathrm{SH}$ in pregnancy progress to overt hypothyroidism? How often should patients be monitored?

Given that SH has been reported to be relatively common in an unselected healthy cohort of women in Casey et al, the cost benefit of a screening programme should be evaluated. The authors speculate that previously documented reduction in $\mathrm{IQ}$ of children born to mothers with $\mathrm{SH}$ may be related to an effect of prematurity. While prematurity can be regarded as a confounder, it is not clear that it can account for the documented changes in neuropsychological development in children of mothers who experienced SH during pregnancy, and requires further evaluation. Home environment has previously been shown to have a profound effect on child neurobehaviour, counteracting the influence of developmental exposures associated with neurodevelopmental impairments. ${ }^{2}$ It will be important to know if mothers with $\mathrm{SH}$ experience any neuropsychiatric impairments.

Warren G Foster, PhD McMaster University Hamilton, Ontario, Canada

1 Lao T. Thyroid disorders in pregnancy. Curr Opin Obstet Gynecol 2005;17:123-7.

2 Walkowiak J, Wiener JA, Fastabend A, et al. Environmental exposure to polychlorinated biphenyls and quality of the home environment: effects on psychodevelopment in early childhood. Lancet 2001;358:1602-7. 